\title{
FLOQUET PROBLEM AND CENTER MANIFOLD \\ REDUCTION FOR ORDINARY DIFFERENTIAL OPERATORS WITH PERIODIC COEFFICIENTS IN HILBERT SPACES
}

Kozlov, Vladimir

2021-05-11

Kozlov , V \& Taskinen , J 2021, ' FLOQUET PROBLEM AND CENTER MANIFOLD

REDUCTION FOR ORDINARY DIFFERENTIAL OPERATORS WITH PERIODIC

COEFFICIENTS IN HILBERT SPACES ' , St Petersburg mathematical journal , vol. 32 , no.

3 , pp. 531-550 . https://doi.org/10.1090/spmj/1660

http://hdl.handle.net/10138/340022

https://doi.org/10.1090/spmj/1660

cc_by_nc_nd

acceptedVersion

Downloaded from Helda, University of Helsinki institutional repository.

This is an electronic reprint of the original article.

This reprint may differ from the original in pagination and typographic detail.

Please cite the original version. 


\title{
FLOQUET PROBLEM AND CENTER MANIFOLD REDUCTION FOR ORDINARY DIFFERENTIAL OPERATORS WITH PERIODIC COEFFICIENTS IN HILBERT SPACES
}

\author{
VLADIMIR KOZLOV ${ }^{1}$, JARI TASKINEN ${ }^{2}$
}

\begin{abstract}
A first order differential equation with a periodic operator coefficient acting in a pair of Hilbert spaces is considered. This setting models both elliptic equations with periodic coefficients in a cylinder and parabolic equations with time periodic coefficients. Our main results are a construction of a pointwise projector and a spectral splitting of the system into a finite dimensional system of ordinary differential equations with constant coefficients and an infinite dimensional part whose solutions have better properties in a certain sense. This complements the well-known asymptotic results for periodic hypoelliptic problems in cylinders [7] and for elliptic problems in quasicylinders [13].

As an application we give a center manifold reduction for a class of non-linear ordinary differential equations in Hilbert spaces with periodic coefficients. This result generalizes the known case with constant coefficients, [10, 11].
\end{abstract}

Keywords: Floquet theorem, differential equations with periodic coefficients, asymptotics of solutions to differential equations, center manifold reduction.

${ }^{1}$ Department of Mathematics, Linköping University, S-581 83 Linköping, Sweden

${ }^{2}$ Department of Mathematics and Statistics, University of Helsinki, P.O.Box 68, 00014 Helsinki, Finland

E-mail: vladimir.kozlov@liu.se/jari.taskinen@helsinki.fi

\section{INTRODUCTION}

Consider a first order ordinary differential equation (ODE) for an unknown function $x(t)$ with values in an infinite dimensional Hilbert space $Y$,

$$
\frac{d x(t)}{d t}=A x(t)+f(t ; x(t)), t \in \mathbb{R},
$$

where $A$ is an unbounded linear operator in $Y$, which is constant in $t$, and $f$ : $\mathbb{R} \times D_{A} \rightarrow Y$ is given. If $\mathcal{P}$ is a finite dimensional orthogonal projector in $Y$ which commutes with $A$, then the system (1.1) with $f \equiv 0$ can be split into a finite dimensional system on the subspace $\mathcal{P}(Y)$ and an infinite dimensional system which may have better properties than the initial one. This reduction can be quite useful in the study of the large time behaviour of linear dynamical systems perturbed by a linear or non-linear perturbation $f$ (see [3], [1] and references there). The main subject of this paper is to study similar splitting for the case when $A=A(t)$ is a periodic operator function with certain Fredholm properties. Our goal is to define a pointwise projector $\mathcal{P}=\mathcal{P}(t)$, which is a projector for any $t \in \mathbb{R}$ and commutes with the operator of the periodic problem. This projector leads to the reduction of the problem to a finite dimensional problem with time independent operator and infinite dimensional problem having better properties than the original one. Another goal is to present a center manifold reduction for nonlinear periodic operators. 
We recall the classical Floquet theorem concerning a system of ODEs

$$
\frac{d x(t)}{d t}=A(t) x(t), t \in \mathbb{R}, x(t) \in \mathbb{R}^{d},
$$

where $A$ is a $d \times d$-matrix depending periodically on $t$. Let $G(t)$ be the fundamental matrix-solution of (1.2). The Floquet theorem says that there exists a constant matrix $C$ and a periodic matrix $P(t)$ such that $G(t)=P(t) e^{C t}$. This theorem allows to reduce the periodic system (1.2) to a system with constant coefficients.

We will consider the infinite dimensional case, where $A$ is an operator in infinite dimensional Hilbert-spaces and depends periodically on $t$. We will use the theory of this equation as developed and exposed in [6] and [7] (see in particular Sect. 5.1, the comments of Ch. 5 and the references there). This allows us to avoid the details of the Floquet-Bloch techniques, on which the theory is based (for a recent presentation of the Floquet-Bloch techniques, see [8]). We also mention the related theory of elliptic problems in periodic quasicylinders, see [13] and [14], although we stick to cylindrical domains here.

Our contribution to the infinite dimensional, periodic problem will consist of a construction of a projection operator and a spectral splitting of the problem as described in the beginning. We also mention the references [3], 4] and [5], the first two of which contain an analogous theory in the case $A(t)$ is a perturbation of an operator $A_{0}$ independent of $t$. We will use the same formalism of analytic Fredholm operator in the way as it is presented in the appendix of [3]. In particular in the treatment of the infinite dimensional part of the splitted system we use a technique developed [3], which allows us to avoid a choice of function spaces for estimating the remainder terms, since all of them can be treated from this "pointwise estimate", see Sect. 4 and 7 in [3].

The new aspects of the above approach are motivated by the possibility to generalize the existing center manifold reduction results of non-linear Hilbert-space valued ODEs to the case of periodic equations. Indeed, in the last section of the paper we will present such an application, where we consider small solutions of a non-linear problem $D_{t} u-A(t) u=f(t, u)$ and present its reduction to a finite dimensional dynamical system, similarly to the constant coefficient case considered by Mielke in [10.

The structure of the paper is the following. In Sect.2 we formulate the periodic infinite dimensional problem, introduce the function spaces and present the main assumptions on the operator of the problem. In Sect. 3 we remind some basic definitions and properties of the eigenvalues, eigenvectors and generalized eigenvectors of the operator pencils associated with our periodic problem. In Sect.4 we collect known results ([7], Ch.4 and 5) on the solvability and asymptotics of solutions to periodic problems. These results are proved in [13] in the case of elliptic boundary value problems with periodic coefficients in periodic cylinders. Our main results are contained in Sect. 5 and [6. In Sect. 5 we introduce a certain operator $\mathcal{P}$ which is actually a projector which commutes both with the periodic operator as well as the point evaluation operator $f \mapsto f(t)$ for all $t$, and delivers a finite dimensional ODE with constant coefficients for $\mathcal{P} u$, where $u$ is a solution to the infinite dimensional system. The main result of our paper is Theorem 6.1 in Sect.6, which gives a splitting of the periodic operator valued equation into a finite dimensional system of ODEs with constant coefficients and an infinite dimensional part whose solutions 
have better properties in a certain sense. In Section 7 we present the main application of Theorem 6.1, namely an extension of the center manifold theorem for ODE in Hilbert space obtained by Mielke in [10], [11], to the periodic coefficient case.

\section{Statement of the Problem}

The setting of our problem is similar to that of [7], Section 5.1, and [3], Part III, except that in the latter, the nature of the $t$-dependence of the operator $A(t)$ is different. All the results of this section are known, see in particular Ch. 1 of the first above mentioned citation. Some proofs are reproduced for the convenience of the reader.

To proceed with details, we let $X$ and $Y$ be Hilbert spaces over the complex scalar field $\mathbb{C}$ such that $X$ is compactly and densely embedded in $Y$. We denote the norms in $X$ and $Y$ by $\|\cdot\|_{X}=\|\cdot ; X\|$ and $\|\cdot\|_{Y}=\|\cdot ; Y\|$, respectively. We identify $Y^{*}$ with $Y$ by using the inner product $(\cdot, \cdot)=(\cdot, \cdot)_{Y}$ and introduce for $h \in Y$ the norm

$$
\|h\|_{X^{*}}=\sup \left\{|(g, h)|: g \in X,\|g\|_{X}=1\right\} .
$$

The completion of $Y$ with respect to this norm coincides with $X^{*}$, and the sesquilinear form $(g, h)$ can be extended for $g \in X$ and $h \in X^{*}$ such that the inequality $|(g, h)| \leq\|g\|_{X}\|h\|_{X^{*}}$ holds. Clearly, $Y \subset X^{*}$.

Given $a, b \in \mathbb{R}, a<b$, we denote by $\mathcal{X}(a, b)$ the space of functions $u:(a, b) \mapsto X$ such that the weak $t$-derivatives with values in $Y$ exist and are locally integrable (in the standard Bochner sense, see e.g. [1], Sect.3.7.) and such that the norm

$$
\|u ; \mathcal{X}(a, b)\|=\left(\int_{a}^{b}\left(\|u(t) ; X\|^{2}+\left\|D_{t} u(t) ; Y\right\|^{2}\right) d t\right)^{1 / 2}
$$

is finite. Here and elsewhere $D_{t}=\partial / \partial t$. Also, the space $\mathcal{Y}(a, b)$ consists of locally integrable functions $u:(a, b) \mapsto Y$ with finite norm

$$
\|f ; \mathcal{Y}(a, b)\|=\left(\int_{a}^{b}\|f(t) ; Y\|^{2} d t\right)^{1 / 2} .
$$

The space $\mathcal{Y}_{\text {loc }}:=L_{\text {loc }}^{2}(\mathbb{R} ; Y)$ consists of measurable functions defined on $\mathbb{R}$ with values in $Y$ with finite semi-norms (2.2) for all $a<b$, and the space $\mathcal{X}_{\text {loc }}:=L_{\text {loc }}^{2}(\mathbb{R} ; X)$ is defined analogously (cf. above); in particular for every $f \in \mathcal{X}_{\text {loc }}$, the semi-norms (2.1) are finite for all $a<b$.

Given $\beta \in \mathbb{R}$, the space $\mathcal{X}_{\beta}$ consists of functions $u \in L_{\text {loc }}^{2}(\mathbb{R} ; X)$ such that $D_{t} u \in$ $L_{\text {loc }}^{2}(\mathbb{R} ; Y)$ and the norm

$$
\left\|u ; \mathcal{X}_{\beta}\right\|=\left(\int_{\mathbb{R}} e^{2 \beta t}\left(\|u(t) ; X\|^{2}+\left\|D_{t} u(t) ; Y\right\|^{2}\right) d t\right)^{1 / 2} \text { is finite, }
$$

and the space $\mathcal{Y}_{\beta}=L_{\beta}^{2}(\mathbb{R} ; Y)$ consists of functions $f \in L_{\text {loc }}^{2}(\mathbb{R} ; Y)$ with finite norm

$$
\left\|f ; \mathcal{Y}_{\beta}\right\|=\left(\int_{\mathbb{R}} e^{2 \beta t}\|f(t) ; Y\|^{2} d t\right)^{1 / 2} .
$$

In order to deal with periodic problems we follow [12, [14] and also introduce subspaces of $\mathcal{X}_{\mathrm{loc}}$ and $\mathcal{Y}_{\mathrm{loc}}$, which consist of periodic functions in $t$ of period 1 and 
which are denoted by $\widehat{\mathcal{X}}$ and $\widehat{\mathcal{Y}}$, respectively. The norms in these spaces are

$$
\|u ; \widehat{\mathcal{X}}\|=\|u ; \mathcal{X}(0,1)\|,\|f ; \widehat{\mathcal{Y}}\|=\|f ; \mathcal{Y}(0,1)\| .
$$

Let $A(t)$ be a bounded operator from $X$ into $Y$ depending continuously on $t \in \mathbb{R}$ with respect to the operator norm. We assume that $A(t)$ is periodic with respect to $t$ with the period 1 . For every $t$, we denote by $A(t)^{*}: Y \rightarrow X^{*}$ the adjoint operator with respect to the duality $(\cdot, \cdot)$, i.e.,

$$
(A(t) \varphi, \psi)=\left(\varphi, A(t)^{*} \psi\right) \quad \varphi \in X, \psi \in Y .
$$

We also define the differential operators

$$
\mathcal{L}=\mathcal{L}\left(t, D_{t}\right):=D_{t} u(t)+A(t) u(t) \text { and } \mathcal{L}^{*}\left(t, D_{t}\right):=-D_{t} u(t)+A(t)^{*} u(t) .
$$

In the following we will consider the problem

$$
\mathcal{L}\left(t, D_{t}\right) u=f(t)
$$

where $f \in L_{\text {loc }}^{2}(\mathbb{R} ; Y)$ is a given function and $u \in \mathcal{X}_{\text {loc }}$ is a function to be found. Our aim is to introduce a reduction of this problem into a system consisting of a scalar valued, finite dimensional ODE-system and of another vector valued ODE, which has better properties then the initial problem. The first main assumptions on $\mathcal{L}$ is the following local estimate (cf. [4], Sect. 2.2)

$$
\begin{aligned}
\|u ; \mathcal{X}(0,1)\| \leq & C\left(\left\|\mathcal{L}\left(t, D_{t}\right) u ; \mathcal{Y}(-1,2)\right\|\right. \\
& +\|u ; \mathcal{Y}(-1,2)\|) \quad \text { for all } u \in \mathcal{X}(-1,2) .
\end{aligned}
$$

To formulate the second assumption let us consider the following operator depending on a complex parameter $\lambda$,

$$
\mathcal{A}(\lambda)=\mathcal{L}\left(t, D_{t}\right)+\lambda: \widehat{\mathcal{X}} \rightarrow \widehat{\mathcal{Y}}, \lambda \in \mathbb{C} .
$$

(Here, for the values $\lambda=i \xi$, the number $\xi \in \mathbb{R}$ would correspond the Floquet parameter or quasimomentum in the Floquet-Bloch transform, but we do not need to exploit this machinery, as mentioned in the introduction.) Obviously, $\mathcal{A}$ is a holomorphic operator pencil with respect to the parameter $\lambda$. The second main assumptions on $\mathcal{L}$ reads as (cf. [12]):

$$
\text { there exists } \lambda_{0} \text { for which } \mathcal{A}\left(\lambda_{0}\right): \widehat{\mathcal{X}} \rightarrow \widehat{\mathcal{Y}} \text { is an isomorphism. }
$$

Remark. We have in mind some applications to parabolic and elliptic PDEproblems, which have been transformed into first order ODE-systems with respect to one of the variables in a canonical way. The assumptions (2.8), (2.10) are natural for such cases. The assumptions would in general fail for hyperbolic PDE-problems.

Lemma 2.1. If the assumptions (2.8) and (2.10) hold, then the families

$$
\begin{aligned}
& \mathcal{A}(\lambda): \mathbb{C} \rightarrow \mathcal{L}(\widehat{\mathcal{X}}, \widehat{\mathcal{Y}}), \\
& \mathcal{A}^{*}(\lambda):=\mathcal{A}(\bar{\lambda})^{*}: \mathbb{C} \rightarrow \mathcal{L}\left(\widehat{\mathcal{Y}}, \widehat{\mathcal{X}}^{*}\right),
\end{aligned}
$$

where $\mathcal{A}^{*}(\lambda)=-D_{t}+A(t)^{*}+\bar{\lambda}$, are holomorphic Fredholm families. Moreover, there holds

$$
\left(\int_{0}^{1}\left(\|u(t) ; X\|^{2}+\left\|\left(D_{t}+\lambda\right) u(t) ; Y\right\|^{2}\right) d t\right)^{1 / 2} \leq C e^{|\Re \lambda|}(\|\mathcal{A}(\lambda) u ; \widehat{\mathcal{Y}}\|+\|u ; \widehat{\mathcal{Y}}\|)
$$


for $u \in \widehat{\mathcal{X}}$. $\widehat{\mathcal{Y}}$.

Here, $\mathcal{L}(\widehat{\mathcal{X}}, \widehat{\mathcal{Y}})$ denotes the Banach space of bounded linear operators from $\widehat{\mathcal{X}}$ into

Proof. Writing estimate (2.8) for the function $e^{\lambda t} u, u \in \widehat{\mathcal{X}}$, we get

$$
\begin{aligned}
& \left(\int_{0}^{1} e^{2 t \Re \lambda}\left(\|u(t) ; X\|^{2}+\left\|\left(D_{t}+\lambda\right) u(t) ; Y\right\|^{2}\right) d t\right)^{1 / 2} \\
& \left.\leq C\left(\left(\int_{-1}^{2} e^{2 t \Re \lambda}\left\|\mathcal{L}\left(t, D_{t}+\lambda\right) u(t) ; Y\right\|^{2} d t\right)^{1 / 2}+\int_{-1}^{2} e^{2 t \Re \lambda}\|u(t) ; Y\|^{2} d t\right)^{1 / 2}\right),
\end{aligned}
$$

which implies estimate (2.11). Since the inclusion $X \subset Y$ is compact, we can use the argument in [9], p. 20 or Theorem 2.1. to see that the embedding $\widehat{\mathcal{X}} \subset \widehat{\mathcal{Y}}$ is also compact. Hence, estimate (2.11) implies that the kernel of $\mathcal{A}(\lambda)$ is finite dimensional and the image is closed for all $\lambda$. This together with assumption (2.10) gives that the operator pencil is Fredholm with the index 0 for all $\lambda$ (see [3], Section A.8).

The definition of the adjoint holomorphic family $\mathcal{A}^{*}(\lambda)$ is as in [3], Section A.9, and its Fredholm property follows from that of the family $\mathcal{A}(\lambda)$, as explained in the citation; see also the next lemma.

Let us provide a description of the dual space $\widehat{\mathcal{X}}^{*}$. To this end we use the inner product to identify $\widehat{\mathcal{Y}}^{*}=\widehat{\mathcal{Y}}$, and we also denote by $L_{\text {per }}^{2}\left(\mathbb{R} ; X^{*}\right)$ the subspace of $L_{\text {loc }}^{2}\left(\mathbb{R} ; X^{*}\right)$ consisting of periodic functions $f: \mathbb{R} \rightarrow X^{*}$, endowed with the norm

$$
\left\|f ; L_{\mathrm{per}}^{2}\left(\mathbb{R} ; X^{*}\right)\right\|=\left(\int_{0}^{1}\left\|f(t) ; X^{*}\right\|^{2} d t\right)^{1 / 2} .
$$

We skip the standard proof of the following lemma.

Lemma 2.2. Under the dual pairing

$$
(u, v)_{\widehat{\mathcal{Y}}}:=\int_{0}^{1}(u(t), v(t)) d t
$$

the dual space $\widehat{\mathcal{X}}^{*}$ of $\widehat{\mathcal{X}}$ consists of periodic functions $w$ represented as

$$
w=w_{0}+D_{t} w_{1}
$$

where $w_{0} \in L_{\mathrm{per}}^{2}\left(\mathbb{R} ; X^{*}\right)$ and $w_{1} \in \widehat{\mathcal{Y}}$, and it is endowed with the norm

$$
\left\|w ; \widehat{\mathcal{X}}^{*}\right\|=\inf \left(\left\|w_{0} ; L_{\text {per }}^{2}\left(\mathbb{R} ; X^{*}\right)\right\|+\left\|D_{t} w_{1} ; \widehat{\mathcal{Y}}\right\|\right)
$$

where the infimum is taken over all representations (2.12). The adjoint $\mathcal{A}^{*}(\lambda)$ of the operator $\mathcal{A}(\lambda)$ satisfies

$$
(\mathcal{A}(\lambda) \varphi, \psi)_{\widehat{\mathcal{Y}}}=\left(\varphi, \mathcal{A}^{*}(\lambda) \psi\right)_{\widehat{\mathcal{Y}}} \quad \varphi \in \widehat{\mathcal{X}}, \psi \in \widehat{\mathcal{Y}}
$$

We end this section with one more lemma. One can easily verify that

$$
e^{2 \pi i t} \mathcal{A}(\lambda) u=\mathcal{A}(\lambda-2 \pi i)\left(e^{2 \pi i t} u\right)
$$

for $u \in \widehat{\mathcal{X}}$. 
Lemma 2.3. Assume that the operator $\mathcal{A}(\mu)$ is an isomorphism for $\mu=\beta+i \xi$ with a fixed $\beta \in \mathbb{R}$ and for all $\xi \in[0,2 \pi)$. Then, for all $u \in \widehat{\mathcal{X}}$ and $\lambda=\beta+i \xi$ with $\xi \in \mathbb{R}$,

$$
\left(\int_{0}^{1}\left(\|u(t) ; X\|^{2}+\left\|\left(D_{t}+\lambda\right) u(t) ; Y\right\|^{2}\right) d t\right)^{1 / 2} \leq C\|\mathcal{A}(\lambda) u ; \widehat{\mathcal{Y}}\|,
$$

where $C$ may depend on $\beta$ but it is independent of $\xi$.

Proof. By (2.13) the optimal constant $c$ in the inequality

$$
\|\mathcal{A}(\lambda) u ; \widehat{\mathcal{Y}}\| \geq c\|u ; \widehat{\mathcal{Y}}\|
$$

is the same for $\lambda$ and $\lambda-2 \pi k i$ for all $k= \pm 1, \pm 2, \ldots$. This together with (2.13) and the assumption of the lemma implies existence of a constant $c_{0}$ such that (2.15) is true for all $\lambda=\beta+i \xi$ with $\xi \in \mathbb{R}$. Using (2.15) we derive (2.14) from (2.11).

\section{Eigenvectors, Generalized eigenvectors, Jordan CHAins}

We recall some basic facts concerning the spectrum of the operator pencil $\mathcal{A}(\lambda)$, (2.9) . For the sake of the presentation, we recall some proofs and otherwise refer to the Appendix [3] for a quick introduction to the topic. As in standard spectral theory of linear operators, the spectrum is the set of those $\lambda \in \mathbb{C}$ such that $\mathcal{A}(\lambda): \widehat{\mathcal{X}} \rightarrow \widehat{\mathcal{Y}}$ is not invertible; $\lambda$ is an eigenvalue, if the kernel of $\mathcal{A}(\lambda)$ is not $\{0\}$.

Since $\mathcal{A}(\lambda): \widehat{\mathcal{X}} \rightarrow \widehat{\mathcal{Y}}$ is a holomorphic Fredholm family and due to the assumption (2.10), the spectrum of $\mathcal{A}(\lambda)$ consists of isolated eigenvalues of finite algebraic multiplicity, see Proposition A.8.4 of [3]. From the relation (2.13) it follows that if $\lambda$ is an eigenvalue then the same is true for $\lambda+2 \pi i$ and their multiplicities coincide. In the following we denote for all $\beta \in \mathbb{R}$

$$
\delta_{\beta}=\{\lambda \in \mathbb{C}: \Re \lambda=\beta, \Im \lambda \in[0,2 \pi)\},
$$

and we choose real numbers

$$
\beta_{1}<\beta_{2}
$$

such that there are no eigenvalues of $\mathcal{A}(\lambda)$ on the the intervals $\delta_{\beta_{1}}$ and $\delta_{\beta_{2}}$. We denote eigenvalues of $\mathcal{A}(\lambda)$ in the set

$$
\left\{\lambda=\beta+i \xi ; \beta_{1}<\beta<\beta_{2}, \xi \in[0,2 \pi)\right\}
$$

by $\lambda_{k}, k=1, \ldots, N$, and let $J_{k}$ and $m_{k, 1}, \ldots, m_{k, J_{k}}$ be the geometric and partial multiplicities of $\lambda_{k}$. Assume that for every $k=1, \ldots, N$,

$$
\varphi_{j, m}^{k}, m=0, \ldots, m_{k, j}-1, j=1, \ldots, J_{k},
$$

is a canonical system of Jordan chains of the linear pencil $\mathcal{A}(\lambda)$ corresponding to $\lambda_{k}$ (see [3], Definition A.4.3, Propositions A.4.4, A.4.5.). The functions

$$
\varphi_{j, 0}^{k}, j=1, \ldots, J_{k}
$$

form a linearly independent sequence of eigenvectors corresponding to the eigenvalue $\lambda_{k}$, while the functions (3.4) with $m \geq 1$ are associated vectors satisfying

$$
\mathcal{A}\left(\lambda_{k}\right) \varphi_{j, 0}^{k}=0, \quad \mathcal{A}\left(\lambda_{k}\right) \varphi_{j, m}^{k}=-\varphi_{j, m-1}^{k}, \quad m=1, \ldots, m_{k, j}-1 .
$$


In the same way, the eigenfunctions and generalized eigenfunctions of the adjoint operator are the solutions of the equations

$$
\mathcal{A}^{*}\left(\lambda_{k}\right) \psi_{j, 0}^{k}=0, \quad \mathcal{A}^{*}\left(\lambda_{k}\right) \psi_{j, m}^{k}=-\psi_{j, m-1}^{k}, \quad m=1, \ldots, m_{k, j}-1 .
$$

It will be important to specify the choice of the functions (3.5) and (3.7) such that certain orthogonality relations are satisfied. Notice that we consider a finite set of eigenvalues, which is fixed by the choice of the numbers $\beta_{1}, \beta_{2}$ above. The following assertion is known and its proof can be found in Remark A.10.3 in [3] (see formula (A.60) there).

Lemma 3.1. If the Jordan chains (3.4) are fixed, then there exist uniquely defined Jordan chains of the adjoint pencil $\mathcal{A}^{*}(\lambda)$ corresponding to the eigenvalue $\overline{\lambda_{k}}$

$$
\psi_{j, m}^{k}, m=0, \ldots, m_{k, j}-1, j=1, \ldots, J_{k},
$$

such that in addition to all equations (3.6) and (3.7) also the following hold true:

$$
\left(\varphi_{j, m_{k, j}-1}^{k}, \psi_{J, m}^{k}\right)_{\hat{\mathcal{Y}}}=\delta_{j}^{J} \delta_{0}^{m}, m=0, \ldots, m_{k, j}-1 .
$$

From now on we assume that the eigenfunctions and generalized eigenfunctions satisfy (3.9). The last relation implies some more orthogonality relations.

Lemma 3.2. Let the Jordan chains (3.4) and (3.8) be the same as in Lemma 3.1. Then the following biorthogonality relations hold:

$$
\left(\varphi_{j, m}^{k}, \psi_{J, m_{K, J}-1-M}^{K}\right)_{\widehat{\mathcal{Y}}}=\delta_{k}^{K} \delta_{j}^{J} \delta_{m}^{M}
$$

for all $k, K, j, J, m, M$.

Proof. Let first $K=k$. Then (3.10) for $m=m_{k, j}-1$ follows from (3.9).

Next we observe that for $m=1, \ldots, m_{k, j}-1$ and $M=1, \ldots, m_{k, J}-1, J=$ $1, \ldots, J_{k}$, the relations (3.6) and (3.7) yield

$$
\begin{aligned}
& \left(\varphi_{j, m}^{k}, \psi_{J, m_{k, J}-1-M}^{k}\right)_{\widehat{\mathcal{Y}}}=-\left(\varphi_{j, m}^{k}, \mathcal{A}^{*}\left(\lambda_{k}\right) \psi_{J, m_{k, J}-M}^{k}\right)_{\widehat{\mathcal{Y}}} \\
= & -\left(\mathcal{A}\left(\lambda_{k}\right) \varphi_{j, m}^{k}, \psi_{J, m_{k, J}-M}^{k}\right)_{\widehat{\mathcal{Y}}}=\left(\varphi_{j, m-1}^{k}, \psi_{J, m_{k, J}-M}^{k}\right)_{\hat{\mathcal{Y}}} .
\end{aligned}
$$

Applying this relation with $m=m_{k, j}-1$ and $M=1, \ldots, m_{k, J}-1$ and using that (3.10) is proved for $m=m_{k, j}-1$, we arrived at (3.10) for $m=m_{k, j}-2$ and $M=0, \ldots, m_{K, J}-2$. Since the relations

$$
\left(\varphi_{j, m}^{k}, \psi_{J, 0}^{k}\right)_{\widehat{\mathcal{Y}}}=0, \quad m=0, \ldots, m_{k, j}-2,
$$

follow from the solvability of (3.6), we arrive at (3.10) for $m=m_{k, j}-2$ and all $M$. Repeating this argument we prove (3.10) for all $m$ and $M$.

We finally show that if $k \neq K$, then the orthogonality in (3.10) automatically holds. For the two eigenfunctions we get the orthogonality $\left(\varphi_{j, 0}^{k}, \psi_{J, 0}^{K}\right)_{\widehat{\mathcal{Y}}}=0$ for all $j=1, \ldots, J_{k}, J=1, \ldots, J_{K}$ by the simple classical argument, since the eigenvalues $\lambda_{k}$ and $\lambda_{K}$ are different. Then, we have, for all $M=0, \ldots, m_{K, J}-2$, all $j, J$,

$$
\begin{aligned}
& \left(\varphi_{j, 0}^{k}, \psi_{J, M}^{K}\right)_{\widehat{\mathcal{Y}}}=-\left(\varphi_{j, 0}^{k}, \mathcal{A}^{*}\left(\lambda_{K}\right) \psi_{J, M+1}^{K}\right)_{\widehat{\mathcal{Y}}}=-\left(\mathcal{A}\left(\lambda_{K}\right) \varphi_{j, 0}^{k}, \psi_{J, M+1}^{K}\right)_{\widehat{\mathcal{Y}}} \\
= & -\left(\mathcal{A}\left(\lambda_{k}\right) \varphi_{j, 0}^{k}, \psi_{J, M+1}^{K}\right)_{\widehat{\mathcal{Y}}}+\left(\lambda_{k}-\lambda_{K}\right)\left(\varphi_{j, 0}^{k}, \psi_{J, M+1}^{K}\right)_{\widehat{\mathcal{Y}}} \\
= & \left(\lambda_{k}-\lambda_{K}\right)\left(\varphi_{j, 0}^{k}, \psi_{J, M+1}^{K}\right)_{\hat{\mathcal{Y}}},
\end{aligned}
$$


where the coefficient $\lambda_{k}-\lambda_{K}$ is non-zero, so that the orthogonality $\left(\varphi_{j, 0}^{k}, \psi_{J, M+1}^{K}\right)_{\hat{\mathcal{y}}}=$ 0 for all $M=0, \ldots, m_{K, J}-1$ and $j, J$ follows by induction. In the same way one obtains $\left(\varphi_{j, m}^{k}, \psi_{J, 0}^{K}\right)_{\hat{\mathcal{y}}}=0$ for all $m=0, \ldots, m_{k, j}-1$ and $j, J$.

Then, one proves the following formulas in the same way as (3.12)

$$
\left(\varphi_{j, m}^{k}, \psi_{J, M}^{K}\right)_{\hat{\mathcal{Y}}}=\left\{\begin{array}{c}
\left(\varphi_{j, m+1}^{k}, \psi_{J, M-1}^{K}\right)_{\hat{\mathcal{Y}}}+\left(\lambda_{k}-\lambda_{K}\right)\left(\varphi_{j, m+1}^{k}, \psi_{J, M}^{K}\right)_{\widehat{\mathcal{Y}}} \\
\left(\varphi_{j, m-1}^{k}, \psi_{J, M+1}^{K}\right)_{\hat{\mathcal{Y}}}+\left(\lambda_{k}-\lambda_{K}\right)\left(\varphi_{j, m}^{k}, \psi_{J, M+1}^{K}\right)_{\widehat{\mathcal{Y}}} .
\end{array}\right.
$$

One can then proceed by induction to get the orthogonality for all indices.

Let us still introduce some more notation with the help of the above introduced Jordan chains: we define

$$
\Phi_{j, m}^{k}(t)=e^{\lambda_{k} t} \sum_{n=0}^{m} \frac{t^{n}}{n !} \varphi_{j, m-n}^{k}=e^{\lambda_{k} t} \sum_{n=0}^{m} \frac{t^{m-n}}{(m-n) !} \varphi_{j, n}^{k},
$$

for all $k=1, \ldots, N, j=1, \ldots, J_{k}, m=0, \ldots, m_{k, j}-1$. It is known and one can verify it directly that these functions are solutions to the homogeneous equation (2.7). The binomial formula implies the following relation which will be needed later:

$$
\begin{aligned}
& e^{\lambda_{k} t} \sum_{n=0}^{m} \frac{(t-\tau)^{n}}{n !} \varphi_{j, m-n}^{k}(t)=e^{\lambda_{k} t} \sum_{n=0}^{m} \sum_{\nu=0}^{n} \frac{t^{n-\nu}(-\tau)^{\nu}}{(n-\nu) ! \nu !} \varphi_{j, m-n}^{k}(t) \\
= & e^{\lambda_{k} t} \sum_{\nu=0}^{m} \frac{(-\tau)^{\nu}}{\nu !} \sum_{n=0}^{m-\nu} \frac{t^{m-\nu-n}}{(m-\nu-n) !} \varphi_{j, n}^{k}(t)=\sum_{\nu=0}^{m} \frac{(-\tau)^{\nu}}{\nu !} \Phi_{j, \nu}^{k}(t) .
\end{aligned}
$$

\section{Some Results on Solvability AND ASymptotics For Problem (2.7)}

We will need some more solvability and asymptotical results for problem (2.7), which are proved for general boundary value problems with periodic coefficients in a cylinder in [7, Sect. 4.2, 5.1, 5.4 (for the case of a periodic quasi-cylinder, see also [13]).

Theorem 4.1. The mapping

$$
\mathcal{L}\left(t, D_{t}\right): \mathcal{X}_{-\beta} \rightarrow \mathcal{Y}_{-\beta}
$$

is isomorphic if the semi-interval $\delta_{\beta}$ does not contain eigenvalues of the operator pencil $\mathcal{A}(\lambda)$.

Theorem 4.2. Let $\beta_{1}<\beta_{2}$ be real numbers such that the semi-intervals $\delta_{\beta_{1}}$ and $\delta_{\beta_{2}}$ do not contain eigenvalues of the operator pencil $\mathcal{A}(\lambda)$ and let $f \in \mathcal{Y}_{-\beta_{1}} \cap \mathcal{Y}_{-\beta_{2}}$. Denote by $u_{1}$ and $u_{2}$ solutions to the problem (2.7) from the spaces $\mathcal{X}_{-\beta_{1}}$ and $\mathcal{X}_{-\beta_{2}}$ respectively (which exist according to Theorem 4.1). Then

$$
u_{2}-u_{1}=\sum_{k=1}^{N} \sum_{j=1}^{J_{k}} \sum_{m=0}^{m_{k j}-1} c_{j, m}^{k} \Phi_{j, m}^{k}(t)
$$

where the functions $\Phi_{j, m}^{k}, k=1, \ldots, N$, are all the functions (3.13) such that the eigenvalues $\lambda_{k}$ belong to the set

$$
Q\left(\beta_{1}, \beta_{2}\right):=\left\{\lambda=\beta+i \xi: \beta_{1}<\beta<\beta_{2}, \xi \in[0,2 \pi)\right\},
$$

and $c_{j, m}^{k}$ are constants. 
A straightforward consequence of the last theorem is the following uniqueness result.

Corollary 4.3. Let $\beta_{1}$ and $\beta_{2}$ be the same as in Theorem 4.2. If $u \in \mathcal{X}_{\mathrm{loc}}$ is a solution of (2.7) with $f=0$ and

$$
\|u ; \mathcal{X}(t, t+1)\| \leq C e^{\beta_{1} t} \text { for } t \geq 0 \text { and }\|u ; \mathcal{X}(t, t+1)\| \leq C e^{\beta_{2} t} \text { for } t \leq 0
$$

for some positive constant $C$, then

$$
u=\sum_{k=1}^{N} \sum_{j=1}^{J_{k}} \sum_{m=0}^{m_{k, j}-1} c_{j, m}^{k} \Phi_{j, m}^{k}(t)
$$

where $c_{j, m}^{k}$ are constants and $\Phi_{j, m}^{k}$ are all functions (3.13) such that the eigenvalues $\lambda_{k}$ belong to the set (4.2).

Proof. Let $\beta_{1}^{\prime}>\beta_{1}$ and $\beta_{2}^{\prime}<\beta_{2}$ be such that the intervals $\left[\beta_{1}, \beta_{1}^{\prime}\right]$ and $\left[\beta_{2}^{\prime}, \beta_{2}\right]$ do not contain the eigenvalues of the operator pencil $\mathcal{A}(\lambda)$. Let also $\eta=\eta(t)$ be a smooth function of one variable such that $\eta(t)=1$ for $t>1$ and $\eta(t)=0$ for $t>0$. Consider the problem (2.7) with $f=\left(D_{t} \eta\right) u$. This problem has two solutions, $u_{1}=\eta u$ and $u_{2}=(\eta-1) u$. Since

$$
\int_{0}^{\infty} e^{-\beta_{1}^{\prime} t}\left\|u_{1}(t) ; X\right\|^{2} d t \leq C \int_{0}^{\infty} e^{-\beta_{1}^{\prime} t}\|u ; \mathcal{X}(t, t+1)\|^{2} d t \leq C \int_{0}^{\infty} e^{t\left(\beta_{1}-\beta_{1}^{\prime}\right)} d t<\infty
$$

$u_{1}$ belongs to $\mathcal{X}_{-\beta_{1}^{\prime}}$. Similarly, $u_{2}$ belongs to $\mathcal{X}_{-\beta_{2}^{\prime}}$. By Theorem 4.2, $u=u_{2}-u_{1}$ is equal to the right-hand side of (4.1) and we arrive at (4.4).

\section{Pointwise projector.}

Let us introduce the operator

$$
\mathcal{P} u(t)=\sum_{k=1}^{N} \sum_{j=1}^{J_{k}} \sum_{m=0}^{m_{k, j}-1} u_{j, m}^{k}(t) \varphi_{j, m_{k, j}-1-m}^{k}(t)
$$

where

$$
u_{j, m}^{k}(t)=\left(u(t), \psi_{j, m}^{k}(t)\right)
$$

and $t \in \mathbb{R}$. If the inner product $(\cdot, \cdot)$ were replaced by $(\cdot, \cdot)_{\hat{\mathcal{Y}}}$ then by (3.10) the above operator would be the finite dimensional spectral projector for the operator $-\mathcal{L}\left(t, D_{t}\right)$ corresponding to the spectrum in the set (3.3). But here we have only the inner product $(\cdot, \cdot)$ on the cross-section and the coefficients $u_{j, m}^{k}$ are functions of $t$. By definition, the operator $\mathcal{P}$ commutes with the point evaluation operator $\mathcal{Y}_{\text {loc }} \ni f \mapsto f(t)$ for all $t \in \mathbb{R}$. The notation $\mathcal{P}(t)$ means the evaluation of (5.1) at $t$.

The operator $\mathcal{P}$ is well defined on the spaces $\mathcal{Y}_{\text {loc }}$ and $\mathcal{X}_{\text {loc }}$. Since there are only finitely terms in the sums (5.1) and

$$
\left|u_{j, m}^{k}(t)\right| \leq C\|u(t) ; Y\| \text { and }\left|\partial_{t} u_{j, m}^{k}(t)\right| \leq C\left\|\partial_{t} u(t) ; Y\right\|,
$$

the operator $\mathcal{P}$ is bounded as an operator in $\mathcal{X}_{\beta}$ and $\mathcal{Y}_{\beta}$.

In the following theorem we prove that the operator $\mathcal{P}(t)$ is indeed a projector and derive some important properties of $\mathcal{P}$. 
Theorem 5.1. The operator $\mathcal{P}$ has the following properties:

(i) $\mathcal{P}(t)$ is a projector for every $t \in \mathbb{R}$;

(ii) $\mathcal{L} \mathcal{P} u=\mathcal{P} \mathcal{L} u$ for all $u \in \mathcal{X}_{\mathrm{loc}}$;

(iii) there holds

$$
\mathcal{L} \mathcal{P} u=\sum_{k=1}^{N} \sum_{j=1}^{J_{k}} \sum_{m=0}^{m_{k, j}-1}\left(\left(D_{t}-\lambda_{k}\right) u_{j, m}^{k}(t)-u_{j, m-1}^{k}(t)\right) \varphi_{j, m_{k, j}-1-m}^{k}(t),
$$

where it is assumed that $u_{j,-1}^{k}=0$.

The proof of is based on the following, perhaps unexpected fact.

Lemma 5.2. We have for all $k, K=1, \ldots, N, j=1, \ldots, J_{k}, J=1, \ldots, J_{K}$, $m=0, \ldots, m_{k, j}-1, M=0, \ldots, m_{K, J}-1$,

$$
\left(\varphi_{j, m_{k, j}-1-m}^{k}(t), \psi_{J, M}^{K}(t)\right)=\delta_{K}^{k} \delta_{J}^{j} \delta_{M}^{m} \quad \text { for all } t \in \mathbb{R} .
$$

We will give a proof of this lemma at the end of this section and now complete the proof of Theorem 5.1. Indeed, the claim ( $i$ ) follows from (5.4). As for (iii), using (3.6) we have

$$
\begin{aligned}
&\left(D_{t}+A(t)\right) U(t) \\
&= \sum_{k=1}^{N} \sum_{j=1}^{J_{k}} \sum_{m=0}^{m_{k, j}-1}\left(\left(D_{t} u_{j, m}^{k}(t)\right) \varphi_{j, m_{k, j}-1-m}^{k}(t)\right. \\
&\left.+u_{j, m}^{k}(t)\left(D_{t}+A(t)\right) \varphi_{j, m_{k, j}-1-m}^{k}(t)\right) \\
&= \sum_{k=1}^{N} \sum_{j=1}^{J_{k}} \sum_{m=0}^{m_{k, j}-1}\left(\left(D_{t}-\lambda_{k}\right) u_{j, m}^{k}(t) \varphi_{j, m_{k, j}-1-m}^{k}(t)\right. \\
&\left.-u_{j, m}^{k}(t) \varphi_{j, m_{k, j}-m-2}^{k}(t)\right),
\end{aligned}
$$

which implies $($ iii). Finally, we have

$$
\begin{aligned}
& \mathcal{P} \mathcal{L} u=\sum v_{j, m}^{k}(t) \varphi_{j, m_{k, j}-1-m}^{k}(t), \text { where } \\
& v_{j, m}^{k}(t)=\left(\left(D_{t}+A(t)\right) u(t), \psi_{j, m}^{k}(t)\right)=D_{t} u_{j, m}^{k}(t)+\left(u(t),\left(-D_{t}+A(t)^{*}\right) \psi_{j, m}^{k}(t)\right) .
\end{aligned}
$$

Using (5.4) we obtain

$$
\begin{aligned}
v_{j, m}^{k}(t) & =D_{t} u_{j, m}^{k}(t)-\lambda_{k}\left(u(t), \psi_{j, m}^{k}(t)\right)-\left(u(t), \psi_{j, m-1}^{k}(t)\right) \\
& =\left(D_{t}-\lambda_{k}\right) u_{j, m}^{k}(t)-u_{j, m-1}^{k}(t) .
\end{aligned}
$$

which together with $(i i i)$ gives $(i i)$.

Proof of Lemma 5.2. Introduce

$$
I(k, j, m ; K, J, M)(t)=\left(\varphi_{j, m}^{k}(t), \psi_{J, M}^{K}(t)\right) .
$$

We have for all $m, M=0, \ldots, m_{k, j}-1$

$$
D_{t} I(k, j, m ; K, J, M)(t)=\left(D_{t} \varphi_{j, m}^{k}(t), \psi_{J, M}^{K}(t)\right)+\left(\varphi_{j, m}^{k}(t), D_{t} \psi_{J, M}^{K}(t)\right) .
$$

Moreover, by (3.6), (3.7),

$$
\left(D_{t}+A(t)+\lambda_{k}\right) \varphi_{j, m}^{k}+\varphi_{j, m-1}^{k}=0,\left(-D_{t}+A(t)^{*}+\overline{\lambda_{K}}\right) \psi_{J, M}^{K}+\psi_{J, M-1}^{K}=0,
$$


where we must agree that the functions with negative second lower index are zero. Thus,

$$
\begin{aligned}
D_{t} I(k, j, m ; K, J, M)(t)= & -\left(\left(A(t)+\lambda_{k}\right) \varphi_{j, m}^{k}(t)+\varphi_{j, m-1}^{k}(t), \psi_{J, M}^{K}(t)\right) \\
& +\left(\varphi_{j, m}^{k}(t),\left(A(t)^{*}+\overline{\lambda_{K}}\right) \psi_{J, M}^{K}(t)+\psi_{J, M-1}^{K}(t)\right) .
\end{aligned}
$$

After cancellation, we get

$$
\begin{aligned}
& D_{t} I(k, j, m ; K, J, M)(t) \\
= & -\left(\varphi_{j, m-1}^{k}(t), \psi_{J, M}^{K}(t)\right)+\left(\varphi_{j, m}^{k}(t), \psi_{J, M-1}^{K}(t)\right) \\
= & -I(k, j, m-1 ; K, J, M)(t)+I(k, j, m ; K, J, M-1)(t) .
\end{aligned}
$$

$1^{\circ}$. We first prove the case $K=k$ and $J=j \in\left\{1, \ldots, J_{k}\right\}$.

( $i$ ) Let first $m=0$ and $M=0$. Then, the right-hand side of (5.6) is zero by the convention made above, and therefore $D_{t} I(k, j, 0 ; k, j, 0)(t)=0$ and thus $I(k, j, 0 ; k, j, 0)(t)$ does not depend on $t$. By Lemma 3.2 we get for all $t$

$$
I(k, j, 0 ; k, j, 0)(t)=\int_{0}^{1} I(k, j, 0, ; k, j, 0)(\tau) d \tau=\left(\varphi_{j, 0}^{k}, \psi_{j, 0}^{k}\right)_{\widehat{\mathcal{Y}}}=0 .
$$

(ii) We next consider the case $m=0, M=1, \ldots, m_{k, j}-2$. We use induction with respect to $M$ : assume that $I(k, j, 0 ; k, j, M)(t)=0$ for some $M<m_{k, j}-2$ and all $t \in[0,1]$. By $(5.6)$ we get

$$
D_{t} I(k, j, 0 ; k, j, M+1)(t)=I(k, j, 0 ; k, j, M)(t)=0
$$

hence, $I(k, j, 0 ; k, j, M+1)(t)$ is constant with respect to $t$. Integrating this constant as in (5.7) and using Lemma 3.2 yield for all $t$

$$
I(k, j, 0 ; k, j, M)(t)=\left(\varphi_{j, 0}^{k}, \psi_{j, M}^{k}\right)_{\widehat{\mathcal{Y}}}=0 \quad \forall M=1, \ldots, m_{k, j}-2 .
$$

(Note that by Lemma 3.2 the inner product is not zero for $M=m_{k, j}-1$.)

In the same way, using inductively

$$
D_{t} I(k, j, m+1 ; k, j, 0)(t)=I(k, j, m ; k, j, 0)(t)=0
$$

for $m=0, \ldots, m_{k, j}-2$ instead of (5.8) we prove that

$$
I(k, j, m ; k, j, 0)=0 \quad \forall m=1, \ldots, m_{k, j}-2 .
$$

(iii) We next consider the case $m+M \leq m_{k, j}-2$ by using a double induction: assume that for some index $M \geq 0$ with $M \leq m_{k, j}-3$ the equality $I(k, j, m ; k, j, M)=$ 0 has been proven for all $m=0, \ldots, m_{k, j}-M-3$. Then, (5.6) implies, for $m=1, \ldots, m_{k, j}-M-2$,

$$
\begin{aligned}
& D_{t} I(k, j, m ; k, j, M+1) \\
= & -I(k, j, m-1 ; k, j, M+1)+I(k, j, m ; k, j, M) .
\end{aligned}
$$

We can thus proceed by induction with respect to $m$ (using $t$-integration and Lemma 3.2 as above) to get

$$
I(k, j, m ; k, j, M+1)=0 \quad \forall m=0, \ldots, m_{k, j}-M-2 .
$$

Induction with respect to $M$ yields (5.4) for all $m+M \leq m_{k, j}-2$. In both induction procedures we use $(i i)$ for $m=0$ and $M=0$ to start with. 
(iv) Consider $m+M=m_{k, j}-1$. Formula (5.6) and what we have proven until now again imply that for every $m=0,1 \ldots, m_{k, j}-1$ the expression

$$
I\left(k, j, m ; k, j, m_{k, j}-m-1\right)
$$

is a constant, which by $t$ - integration and Lemma 3.2 is equal to 1 .

$(v)$ However, this and (5.6) again imply that for every $m=1,2, \ldots, m_{k, j}-1$ we have

$$
I\left(k, j, m ; k, j, m_{k, j}-m\right)=0 .
$$

(vi) From here on we can continue in the same way as in (iii) to get the result for $M+m \geq m_{k, j}$.

$2^{\circ}$. The proof in the case $K=k$ but $J \neq j$ is simpler than $1^{\circ}$. The case $(i)$ is the same. The argument of the case $(i i)$ yields $I(k, j, m ; k, J, M)=0$ for the pairs $(m, M)$ with $m=0, M=1, \ldots, m_{k, J}-1$ and $m=1, \ldots, m_{k, j}-1, M=0$, by Lemma 3.2. Then, the procedure of (iii) yields $I(k, j, m ; k, J, M)=0$ for all remaining pairs $(m, M)$, since now we do not have the obstruction of the case $(i v)$ (the inner products $\left(\varphi_{j, m}^{k}, \psi_{J, M}^{k}\right)_{\hat{y}}$ equal 0 instead of 1 , by Lemma 3.2, for all indices in question).

$3^{\circ}$. For the proof in the case $K \neq k$ we need to introduce instead of (5.5),

$$
I(k, j, m ; K, J, M)(t)=e^{\left(\lambda_{k}-\lambda_{K}\right) t}\left(\varphi_{j, m}^{k}(t), \psi_{J, M}^{K}(t)\right),
$$

because $\lambda_{k} \neq \lambda_{K}$. By a similar calculation as around (5.6) we get for all $j, J, m, M$

$$
\begin{aligned}
& e^{\left(-\lambda_{k}+\lambda_{K}\right) t} D_{t} I(k, j, m ; K, J, M)(t) \\
= & \left(\lambda_{k}-\lambda_{K}\right)\left(\varphi_{j, m}^{k}(t), \psi_{J, M}^{K}(t)\right) \\
+ & \left(D_{t} \varphi_{j, m}^{k}(t), \psi_{J, M}^{K}(t)\right)+\left(\varphi_{j, m}^{k}(t), D_{t} \psi_{J, M}^{K}(t)\right) \\
= & -\left(\varphi_{j, m-1}^{k}(t), \psi_{J, M}^{K}(t)\right)+\left(\varphi_{j, m}^{k}(t), \psi_{J, M-1}^{K}(t)\right) .
\end{aligned}
$$

The following argument shows that we can use (5.9) instead of (5.6) and repeat the proof of the case $2^{\circ}$ (i.e. the steps $(i)-($ iii $)$ in $1^{\circ}$ ) for all $j, J, m, M$ : assume that the right-hand side of $(5.9)$ equals 0 for all $t$. Since $e^{\left(-\lambda_{k}+\lambda_{K}\right) t} \neq 0$, this implies that $D_{t} I(k, j, m ; K, J, M)(t)=0$ for all $t$, hence, $I(k, j, m ; K, J, M)(t)=B$ for some constant $B$, for all $t$. Thus, by Lemma 3.2.

$$
0=\left(\varphi_{j, m}^{k}, \psi_{J, M}^{K}\right)_{\widehat{\mathcal{Y}}}=\int_{0}^{1} e^{\left(\lambda_{K}-\lambda_{k}\right) t} e^{\left(\lambda_{k}-\lambda_{K}\right) t}\left(\varphi_{j, m}^{k}(t), \psi_{J, M}^{K}(t)\right) d t=B \int_{0}^{1} e^{\left(\lambda_{K}-\lambda_{k}\right) t} d t .
$$

Here we have $\int_{0}^{1} e^{\left(\lambda_{K}-\lambda_{k}\right) t} d t \neq 0$, since $\lambda_{K}-\lambda_{k}$ cannot equal a multiple of $i 2 \pi$, see (3.3). Hence, the constant $B$ must be zero, and thus also $\left(\varphi_{j, m}^{k}(t), \psi_{J, M}^{K}(t)\right)=0$ for all $t$.

\section{Spectral Splitting.}

We assume that $\beta_{1}<\beta_{2}$ are the same real numbers as in Theorem 4.2 so that in particular the semi-intervals $\delta_{\beta_{1}}, \delta_{\beta_{2}}$ do not contain eigenvalues of the pencil $\mathcal{A}(\lambda)$ and its eigenvalues in the set $Q\left(\beta_{1}, \beta_{2}\right)$ of (4.2) are $\lambda_{1}, \ldots, \lambda_{N}$. We introduce the function

$$
\mu(t)=e^{-\beta_{1} t} \text { for } t \geq 0 \text { and } \mu(t)=e^{-\beta_{2} t} \text { for } t \leq 0 .
$$


Using the projector $\mathcal{P}$ of (5.1) we represent a solution of (2.7) as

$$
u=U+V, \text { where } U=\mathcal{P} u, V=\mathcal{Q} u:=(I-\mathcal{P}) u .
$$

Due to the commutation relation Theorem 5.1(ii) we have the following system of equations for $U$ and $V$ :

$$
\begin{aligned}
\mathcal{L}\left(t, D_{t}\right) U(t) & =\left(D_{t}+A(t)\right) U(t)=\mathcal{P} f \\
\mathcal{L}\left(t, D_{t}\right) V(t) & =\left(D_{t}+A(t)\right) V(t)=\mathcal{Q} f .
\end{aligned}
$$

Using representations (5.1), (5.3) together with Theorem 5.1(iii) and writing

$$
\mathcal{P} f(t)=\sum_{k=1}^{N} \sum_{j=1}^{J_{k}} \sum_{m=0}^{m_{k, j}-1} f_{j, m}^{k}(t) \varphi_{j, m_{k, j}-1-m}^{k}(t),
$$

where

$$
f_{j, m}^{k}(t)=\left(f(t), \psi_{j, m}^{k}(t)\right)
$$

we can present (6.1) as a system of first order differential equations

$$
\left(D_{t}-\lambda_{k}\right) u_{j, m}^{k}(t)+u_{j, m-1}^{k}=f_{j, m}^{k},
$$

Here, $k=1, \ldots, N, j=1, \ldots, J_{k}, m=0, \ldots, m_{k, j}-1$, and $u_{j, m}^{k}$ is given by (5.2) and we assume that $u_{j, m-1}^{k}=0$ if $m=0$.

The equation (6.2) concerns the "remainder" term: here we have removed the spectrum $\lambda_{1}, \ldots, \lambda_{N}$ from the operator using the projector, and hence it has better estimates. This property of the equation (6.2) is contained in the following assertion.

Theorem 6.1. Let $f \in L_{\mathrm{loc}}^{2}(\mathbb{R} ; Y)$ and

$$
\int_{\mathbb{R}} \mu(t)\|f ; \mathcal{Y}(t, t+1)\| d t<\infty .
$$

Then, the equation

$$
\mathcal{L}\left(t, D_{t}\right) u=f
$$

has a solution $u=U+V \in \mathcal{X}_{\mathrm{loc}}$ such that $U$ is a solution of (6.1) and $V$ is a solution of (6.2) satisfying the estimate

$$
\|V ; \mathcal{X}(\tau, \tau+1)\| \leq C \int_{\mathbb{R}} \mu(t-\tau)\|\mathcal{Q} f ; \mathcal{Y}(t, t+1)\| d t
$$

for all $\tau \in \mathbb{R}$.

Let $f$ satisfy (6.4) and $\mathcal{Q} f=0$. If the bounds (4.3) with some constant $C$ hold for $u$, then $V=0$.

Proof. We need to prove here the existence of $V$ satisfying (6.5) as well as the last uniqueness statement. We start by the uniqueness. Let $\mathcal{Q} f=0$ and assume $u$ satisfies (4.3). Let the coefficients of $\mathcal{P} u$ in (5.1) satisfy (6.3). Then $\mathcal{P} u$ is a solution to (2.7) and by analysing solutions of the ODEs (6.3) we conclude that this solution also satisfies (4.3) possibly with a slightly larger $\beta_{1}$ and smaller $\beta_{2}$. Then, according to the above uniqueness result from Corollary 4.3 we have $u=\mathcal{P} u$ and hence $V=\mathcal{Q} u=0$. 
Let us turn to the existence. Let first $f$ have a compact support and let $g=\mathcal{Q} f$. Applying Theorem 4.1 to the equation $\mathcal{L} V=g$, we get the estimates

$$
\int_{-\infty}^{\infty} e^{-\beta_{k}(t-\tau)}\|V(t) ; X\|^{2} d t \leq C \int_{-\infty}^{\infty} e^{-\beta_{k}(t-\tau)}\|g(t) ; Y\|^{2} d t
$$

for $k=1,2$ and all $\tau \in \mathbb{R}$. Finally, assume $f$ is as in (6.4) and write $f=\sum_{j=-\infty}^{\infty} f_{j}$, where

$$
f_{j}(t)=f \text { on }(j, j+1) \text { and } f_{j}=0 \text { for } t \text { outside }(j, j+1), j=0, \pm 1, \pm 2, \ldots
$$

Using estimate (6.6) for the function $V_{j}$ (corresponding to $f_{j}$ and $g_{j}=\mathcal{Q} f_{j}$ ) we get

$$
\left(\int_{\tau}^{\tau+1}\left\|V_{j}(t) ; X\right\|^{2} d t\right)^{1 / 2} \leq C \mu(j-\tau)\left(\int_{j}^{j+1}\|\mathcal{Q} f(t) ; Y\|^{2} d t\right)^{1 / 2} .
$$

Summing up these relations, we obtain for all $\tau \in \mathbb{R}$

$$
\left(\int_{\tau}^{\tau+1}\|V(t) ; X\|^{2} d t\right)^{1 / 2} \leq C \int_{\mathbb{R}} \mu(t-\tau)\|\mathcal{Q} f(t) ; Y\| d t,
$$

which is the same as (6.5).

Remark 6.2. We note that the function $\mu(t)$ is the Green function of the second order operator $-\left(D_{t}-\beta_{1}\right)\left(D_{t}-\beta_{2}\right)$ up to a positive constant factor. So the estimate (6.5) is similar to the representation of the solutions to the equation

$$
-\left(D_{t}-\beta_{1}\right)\left(D_{t}-\beta_{2}\right) u(t)=f(t)
$$

through the Green function and the right-hand side.

Estimates (6.4) and (6.5) imply estimate (4.3) possibly with some slightly larger (smaller) $\beta_{1}\left(\beta_{2}\right)$ and hence uniqueness and existence parts in Theorem 6.1 are in agreement.

\section{ReduCtion of A DYNAMiCAL SYSTEM With PERIODIC COEFFICIENTS IN HILBERT SPACE}

7.1. Formulation of the problem. In this section we apply Theorem 6.1, in a slightly modified form, to obtain a center manifold reduction for a non-linear ODE with periodic coefficients in a Hilbert space. The proofs are mainly quite straightforward modifications of existing result, hence, we only sketch many of them.

To formulate the problem we let the Hilbert spaces $X$ and $Y$ and the operators $A(t)$ and $\mathcal{A}(\lambda)$ be as in Section 2, Then, consider the following non-linear ODE in the space $X$,

$$
\mathcal{L}\left(t, D_{t}\right) u(t)=f(t, \mu, u(t)),
$$

where

$$
f: \mathbb{R} \times \mathcal{M} \times \tilde{X} \rightarrow Y
$$

and $\mathcal{M}$ is the set of parameters, which is a neighborhood of a point $\mu_{0} \in \mathbb{R}^{d}$, while $\tilde{X}$ is a neighborhood of zero in $X$ and $u \in \mathcal{X}_{\text {loc }}$ with $u(t) \in \tilde{X}$ for all $t$. Moreover we assume that the function $f$ of (7.2) belongs to $C_{B U}^{k+1}(\mathbb{R} \times \mathcal{M} \times \tilde{X} ; Y)$ (the space of functions $\mathbb{R} \times \mathcal{M} \times \tilde{X} \rightarrow Y$, which, together with all $t$-, $\mu$ - and $u$-derivatives up to 
order $k+1, k \geq 1$, are uniformly continuous and bounded; by the $u$-derivative we mean the standard Fréchet-derivative in Banach-spaces) and that

$$
f\left(t, \mu_{0}, 0\right)=0, \partial_{u} f\left(t, \mu_{0}, 0\right)=0 \text { for } t \in \mathbb{R} .
$$

Observe that according to these equalities, $f$ is in a sense at least of the second degree with respect to $u$, a property which will be essential to the fixed point argument in the proof of the main result. We assume also that the periodic operator function $A(t)$ belongs to $C_{\text {per }}^{k+1}(\mathbb{R} ; L(X, Y)$ ) (the space of $k+1$ times continuously differentiable functions $\mathbb{R} \rightarrow L(X, Y)$ which are periodic in $t)$.

In this section and in what follows we denote by $\lambda_{1}, \ldots, \lambda_{N}$ the eigenvalues of $\mathcal{A}(\lambda)$ on the interval $\delta_{0}$, (3.1), and for their multiplicities, partial multiplicities, eigenvalues and generalized eigenvalues we keep the notation of 3 . Introduce the set of indices

$$
\Theta=\left\{(k, j, m): k=1, \ldots, N ; j=1, \ldots, J_{k} ; m=0, \ldots, m_{k, j}-1\right\},
$$

and the total algebraic multiplicity of all eigenvalues on the interval $\delta_{0}$

$$
M=\sum_{k=1}^{N} \sum_{j=1}^{J_{k}} m_{k, j}
$$

Following Sections 5, 6] we represent the function $u$ of (7.1) as

$$
u=U+V,
$$

where

$$
U(t)=\mathcal{P}(t) u(t)=\sum_{(k, j, m) \in \Theta} u_{j, m}^{k}(t) \varphi_{j, m_{k, j}-1-m}^{k}(t),
$$

with

$$
u_{j, m}^{k}(t)=\left(u(t), \psi_{j, m}^{k}(t)\right)
$$

and $V=u-U=\mathcal{Q} u, \mathcal{Q}=\mathcal{I}-\mathcal{P}$. Due to additional smoothness of $A(t)$ we have $\varphi_{j, m}^{k} \in C_{\mathrm{per}}^{k}(\mathbb{R} ; X)$ and $\psi_{j, m}^{k} \in C_{\mathrm{per}}^{k}(\mathbb{R} ; Y)$. Now equation (7.1) can be transformed into the system

$$
\begin{aligned}
& \mathcal{L}\left(t, D_{t}\right) U(t)=\mathcal{P} f(t, U(t)+V(t)), \\
& \mathcal{L}\left(t, D_{t}\right) V(t)=\mathcal{Q} f(t, U(t)+V(t)) .
\end{aligned}
$$

By Theorem 5.1, equation (17.7) can be written as the scalar system

$$
\left(D_{t}-\lambda_{k}\right) u_{j, m}^{k}(t)-u_{j, m-1}^{k}(t)=f_{j, m}^{k}(t, U(t)+V(t)), \quad(k, j, m) \in \Theta,
$$

where $u_{j,-1}^{k}=0$ and

$$
f_{j, m}^{k}(t, u(t))=\left(f(t, \mu, u(t)), \psi_{j, m}^{k}(t)\right)
$$

for all $k, j, m$. We introduce the notation

$$
\mathbf{u}(t)=\left\{u_{j, m}^{k}(t)\right\}_{(k, j, m) \in \Theta}=\left\{\left(u(t), \psi_{j, m}^{k}(t)\right)\right\}_{(k, j, m) \in \Theta}, \quad \mathbf{f}=\left\{f_{j, m}^{k}\right\}_{(k, j, m) \in \Theta}
$$

and define the $M \times M$-matrix by

$$
\mathcal{R} \mathbf{u}=\left\{\lambda_{k} u_{j, m}^{k}+u_{j, m-1}^{k}\right\}_{(k, j, m) \in \Theta}
$$

Given $\tau \in \mathbb{R}$ and $\xi \in \mathbb{C}^{M}$ we associate to the equation (7.9) the problem

$$
\left(D_{t}-\mathcal{R}\right) \mathbf{u}(t)(t)=\mathbf{f}(t, \mu, U(t)+V(t)), \mathbf{u}(\tau)=\xi,
$$


where $U$ is expressed through $\mathbf{u}$ by (7.5). This reformulation will replace (7.7) in the later considerations (see (7.35) ) in the general case of complex Hilbert spaces $X$ and $Y$. In the next section we make remarks on the real case.

7.2. Projector $\mathcal{P}$ in the case of real Hilbert spaces. We consider here the case when the operator $A(t)$ is real, or real valued. Namely, in possible applications for partial differential operators having real coefficients, it may very well be desirable to stick to the real valued solutions, but in (17.13), there is no guarantee that for example the matrix $\mathcal{R}$ is a real one. Accordingly, we derive here a modified equation with a real $\mathcal{R}$.

To be precise, we assume here that our basic Hilbert spaces $X$ and $Y$, Section 2 , are presented as a standard complexification of the Hilbert spaces $\widehat{X}, \widetilde{X}, \widehat{Y}$ and $\widetilde{Y}$, respectively, over the real scalar field $\mathbb{R}$, i.e.

$$
X=\widehat{X} \oplus i \widetilde{X} \text { and } Y=\widehat{Y} \oplus i \widetilde{Y}
$$

as vector spaces. Then, $\bar{x}=\widehat{x}-i \widetilde{x}$ denotes the conjugation of an element $x=$ $\widehat{x}+i \widetilde{x} \in \widehat{X} \oplus i \widetilde{X}=X$ (here, $\widehat{x}$ and $\widetilde{x}$ are the real and imaginary parts of $x$ ). Moreover, $x \in X$ is real, if $x \in \widehat{X}$, or equivalently $x=\bar{x}=\widehat{x}$; the notions of the real and imaginary parts of $x$ are as usual. The operator $A(t): X \rightarrow Y$ is real, if there holds $A(t)=\overline{A(t)}$, where $\overline{A(t)}$ is the conjugation of the operator $A(t)$ defined by

$$
\overline{A(t)}(\widehat{x}+i \widetilde{x})=\overline{A(t)(\widehat{x}-i \widetilde{x})} \text { for all } \widehat{x}, \tilde{x} \in X .
$$

If $A(t)$ is real, there holds $A(t)(\widehat{X}) \subset \widehat{Y}$. For example, if $X$ is a Sobolev-space $H^{1}(\Omega)$ on some domain $\Omega \subset \mathbb{R}^{d}$, then the above concepts have natural interpretations in terms of real valued functions and partial differential operators with real coefficients etc.

We introduce the operators $\widehat{P}_{k}: \widehat{\mathcal{Y}} \rightarrow \widehat{\mathcal{Y}}$ as

$$
\widehat{P}_{\lambda_{k}} u=\sum_{j=1}^{J_{k}} \sum_{m=0}^{m_{k, j}-1}\left(u, \psi_{j, m}^{k}\right)_{\widehat{\mathcal{Y}}} \varphi_{j, m_{k, j}-1-m}^{k} .
$$

Let also

$$
\mathcal{P}_{\lambda_{k}}(t) u(t)=\sum_{j=1}^{J_{k}} \sum_{m=0}^{m_{k, j}-1}\left(u(t), \psi_{j, m}^{k}(t)\right)_{Y} \varphi_{j, m_{k, j}-1-m}^{k}(t),
$$

which is now defined for all $u \in C_{\text {loc }}(\mathbb{R} ; Y)$. Clearly, $\widehat{P}_{\lambda_{k}}$ is the spectral projector of the operator pencil $\mathcal{L}$ corresponding to the eigenvalue $\lambda_{k}$ and taking values in the space of periodic functions. Similarly to Lemma 5.2 one can show that the operators $\mathcal{P}_{\lambda_{k}}(t)$ are projectors for every $t \in \mathbb{R}$, i.e. we have $\mathcal{P}_{\lambda_{k}}^{2}=\mathcal{P}_{\lambda_{k}}$. These projectors also have the properties

$$
\mathcal{P}(t)=\sum_{k=1}^{N} \mathcal{P}_{\lambda_{k}}(t) \quad \text { and } \quad \mathcal{P}_{\lambda_{k}}(t) \mathcal{P}_{\lambda_{K}}(t)=\delta_{k, K} \text { for } k, K=1, \ldots, N
$$

According to (2.13), the numbers $\lambda_{k}-2 \pi i$ are also eigenvalues of the pencil $\mathcal{A}(\lambda)$ of the same multiplicity and partial multiplicities as $\lambda_{k}$, and the systems

$$
e^{-2 \pi i} \varphi_{j, m}^{k} \text { and } e^{-2 \pi i} \psi_{j, m}^{k}, \quad j=1, \ldots, J_{k}, m=0, \ldots, k_{k, j}-1,
$$


are canonical Jordan chains corresponding to the eigenvalue $\lambda_{k}-2 \pi i$, and the relation (3.10) holds for them. If the projector $\mathcal{P}_{\lambda_{k}-2 \pi i}(t)$ is defined analogously to (7.14) by using (7.15), then one can verify that actually

$$
\mathcal{P}_{\lambda_{k}-2 \pi i}(t)=\mathcal{P}_{\lambda_{k}}(t)
$$

In the case a real valued operator $A(t)$ the number $\overline{\lambda_{k}}$ is also the eigenvalue of $\mathcal{A}(\lambda)$ of the same multiplicity and partial multiplicities as $\lambda_{k}$. There also holds the important relation

$$
\mathcal{P}_{\overline{\lambda_{k}}}(t)=\overline{\mathcal{P}_{\lambda_{k}}}(t)
$$

It will be convenient to re-numerate eigenvalues as follows. We first consider the eigenvalues $\lambda_{k} \in(0, \pi i)$ and numerate them by $1, \ldots, \sigma$. Then the interval $(\pi i, 2 \pi i)$ contains the same number of eigenvalues since the numbers $\lambda_{k}, \lambda_{k}-2 \pi i$ and $-\lambda_{k}+2 \pi i$ are all eigenvalues of the same multiplicity and partial multiplicities. The eigenvalues in $(\pi i, 2 \pi i)$ are indexed by the numbers $-1, \ldots,-\sigma$ so that

$$
-\lambda_{s}=\lambda_{-s}-2 \pi i
$$

Thus,

$$
\left.\mathcal{P}(t)=\sum_{s=1}^{\sigma}\left(\mathcal{P}_{s}(t)+\overline{\mathcal{P}_{s}}(t)\right)+\left(\epsilon_{0} \mathcal{P}_{0}(t)\right)+\epsilon_{\sigma+1} \mathcal{P}_{\sigma+1}(t)\right)
$$

Here, $\epsilon_{0}=1$, if $2 \pi i$ is an eigenvalue, otherwise $\epsilon_{0}=0$. Similarly, $\epsilon_{\sigma+1}=1$, if $\pi i$ is an eigenvalue, otherwise this coefficient is 0 .

If $2 \pi i$ is an eigenvalue, then due to (7.16) we have $\mathcal{P}_{2 \pi i}(t)=\mathcal{P}_{0}(t)$; one can choose all eigenfunctions and associated eigenfunctions $\varphi_{m, j}^{0}$ and $\psi_{m, j}^{0}$ to be real. Hence, the expression for the operator $\mathcal{P}_{0}(t)$ consists of real terms only for each $t$. If $\lambda=\pi i$ is an eigenvalue of the operator pencil $\mathcal{A}(\lambda)$ then the corresponding projector $\mathcal{P}_{\sigma+1}(t)$ has the form

$$
\left(\mathcal{P}_{\sigma+1} u(t)\right)(t)=\sum_{j=1}^{J_{\sigma+1}} \sum_{m=0}^{m_{\sigma+1, j}} u_{j, m}^{\sigma+1}(t) \varphi_{j, m_{\sigma+1, j}-1-m}^{\sigma+1}(t)
$$

where

$$
u_{j, m}^{\sigma+1}(t)=\left(u(t), \psi_{j, m}^{\sigma+1}(t)\right)_{Y}
$$

and

$$
\varphi_{j, m}^{\sigma+1} \text { and } \psi_{j, m}^{\sigma+1}, \quad j=1, \ldots, J_{\sigma+1}, m=0, \ldots, m_{\sigma+1, j}-1,
$$

are canonical Jordan chains corresponding to the eigenvalue 0 of the operators $D_{t}+$ $A(t)$ and $-D_{t}+A^{*}(t)$ with anti-periodic conditions $(u(t+1)=-u(t))$. The chains are subject to the biorthogonality conditions (3.10). Clearly, the functions (7.19) can be chosen to be real 1 . So the projector $\mathcal{P}(t)$ is real for each $t$.

To give a more explicit representation for $\mathcal{P}(t)$ we write the real and imaginary parts

$$
\varphi_{j, m}^{k}(t)=\widehat{\varphi}_{j, m}^{k}+i \widetilde{\varphi}_{j, m}^{k} \text { and } \psi_{j, m}^{k}(t)=\widehat{\psi}_{j, m}^{k}+i \widetilde{\psi}_{j, m}^{k}
$$

Then for real valued $u$ we have

$$
\mathcal{P}(t) u(t)=\sum_{s=1}^{\sigma} \sum_{j=1}^{J_{s}} \sum_{m=0}^{m_{s, j}-1}\left(\widehat{u}_{j, m}^{s}(t) \widehat{\varphi}_{j, m_{s, j}-1-m}^{s}(t)+\widetilde{u}_{j, m}^{s}(t) \widetilde{\varphi}_{j, m_{s, j}-1-m}^{s}(t)\right)
$$

\footnotetext{
${ }^{1}$ We note that isomorphism between periodic and anti-periodic vector-functions is given by multiplication by $e^{\pi i t}$.
} 


$$
+\sum_{s=0, \sigma+1}^{\sigma} \sum_{j=1}^{J_{s}} \sum_{m=0}^{m_{s, j}-1} \epsilon_{s} \widehat{u}_{j, m}^{s}(t) \widehat{\varphi}_{j, m_{s, j}-1-m}^{s}(t),
$$

where

$$
\widehat{u}_{j, m}^{s}(t)=\left(u(t), \widehat{\psi}_{j, m}^{s}(t)\right)_{Y} \text { and } \widetilde{u}_{j, m}^{s}(t)=\left(u(t), \widetilde{\psi}_{j, m}^{s}(t)\right)_{Y} .
$$

By taking the real and imaginary parts in (7.9) we get the real equations

$$
\begin{aligned}
& D_{t} \widehat{u}_{j, m}^{s}+\mu_{s} \widetilde{u}_{j, m}^{s}-\widehat{u}_{j, m-1}^{s}=\widehat{f}_{j, m}^{s}, \\
& D_{t} \widetilde{u}_{j, m}^{s}-\mu_{s} \widehat{u}_{j, m}^{s}-\widetilde{u}_{j, m-1}^{s}=\widetilde{f}_{j, m}^{s},
\end{aligned}
$$

where $\lambda_{s}=i \mu_{s}$ and

$$
\widehat{f}_{j, m}^{s}(t)=\left(f(t), \widehat{\psi}_{j, m}^{s}(t)\right)_{Y} \text { and } \widetilde{f}_{j, m}^{s}(t)=\left(f(t), \widetilde{\psi}_{j, m}^{s}(t)\right)_{Y} .
$$

In the case $2 \pi i$, or 0 , are also eigenvalues, we get one more equation,

$$
D_{t} \widehat{u}_{j, m}^{0}-\widehat{u}_{j, m-1}^{0}=\widehat{f}_{j, m}^{0},
$$

since $\varphi_{j, m}^{0}, \psi_{j, m}^{0}$ and $f_{j, m}^{0}$ are real, see above. Similarly, if $\pi i$ is an eigenvalue, then there is the additional equation

$$
D_{t} \widehat{u}_{j, m}^{\sigma+1}-\widehat{u}_{j, m-1}^{\sigma+1}=\widehat{f}_{j, m}^{\sigma+1} .
$$

In the decomposition (7.4), i.e. $u(t)=\mathcal{P}(t) u(t)+V(t)$, the projector $\mathcal{P}(t) u(t)$ has the new representation (7.20) and $V$ satisfies the same estimate (6.5) as before. However, the matrix (7.12) can now be replaced by another one, which is obtained from the system (7.21)-(7.25) and has real entries. We keep the old notation and still write system (7.13) as

$$
D_{t} \mathbf{u}-\mathcal{R} \mathbf{u}=\mathbf{f}, \quad \mathbf{u}(\tau)=\xi \in \mathbb{R}^{M},
$$

where $\mathcal{R}$ is a real matrix. In the real case we will use the coordinates

$$
\left(\widehat{\xi}_{j, m}^{s}, \tilde{\xi}_{j, m}^{s}, \widehat{\xi}_{j, m}^{0}, \widehat{\xi}_{j, m}^{\sigma+1}\right), \quad s=1, \ldots, \sigma,
$$

where the last two are there only when $2 \pi i$ or/and $\pi i$ are eigenvalues respectively.

7.3. Model problem. Hereinafter, we formulate all results for the case of real Hilbert spaces. Their generalizations onto complex Hilbert spaces are quite straightforward.

We will need to consider a linearization of the system (7.7)-(7.8). To formulate this we introduce for $\gamma>0$ the weighted Sobolev-type space $\mathbf{H}_{\gamma}^{1}(\mathbb{R})$ of vector functions $\mathbf{u}: \mathbb{R} \rightarrow \mathbb{R}^{M}$ with finite norm

$$
\left\|\mathbf{u} ; \mathbf{H}_{\gamma}^{1}(\mathbb{R})\right\|_{\tau}=\left(\int_{\mathbb{R}} e^{-2 \gamma|t-\tau|}\left(\|\mathbf{u}(t)\|^{2}+\left\|D_{t} \mathbf{u}(t)\right\|^{2} d t\right)^{1 / 2}\right.
$$

(here and in what follows, $\|\cdot\|$ without a Banach space denotes the Euclidean norm of $\mathbb{R}^{M}$ ) and the space $\mathbf{X}_{\gamma}(\mathbb{R})$ consisting of functions $V \in \mathcal{X}_{\text {loc }}$ satisfying $\mathcal{Q}(t) V(t)=$ $V(t)$ for almost all $t \in \mathbb{R}$ with finite norm

$$
\left\|V ; \mathbf{X}_{\gamma}(\mathbb{R})\right\|_{\tau}=\left(\int_{\mathbb{R}} e^{-2 \gamma|t-\tau|}\left(\|V(t) ; X\|^{2}+\left\|D_{t} V(t) ; Y\right\|^{2}\right) d t\right)^{1 / 2}
$$

Given $\tau \in \mathbb{R}$ and $\xi \in \mathbb{R}^{M}$, we pose the following linear problem

$$
\begin{aligned}
\left(D_{t}-\mathcal{R}\right) \mathbf{u}(t) & =\mathbf{F}(t), \quad \mathbf{u}(\tau)=\xi \\
\mathcal{L}\left(t, D_{t}\right) V(t) & =\mathbf{G}(t),
\end{aligned}
$$


for the unknown pair $(\mathbf{u}, V) \in \mathbf{H}_{\gamma}^{1}(\mathbb{R}) \times \mathbf{X}_{\gamma}(\mathbb{R})$. Here, $\mathbf{F}: \mathbb{R} \rightarrow \mathbb{R}^{M}$ is a given vector function belonging to the weighted space $\mathbf{L}_{\gamma}^{2}(\mathbb{R})$ endowed with the norm

$$
\left\|\mathbf{F} ; \mathbf{L}_{\gamma}^{2}(\mathbb{R})\right\|_{\tau}=\left(\int_{\mathbb{R}} e^{-2 \gamma|t-\tau|}\|\mathbf{F}(t)\|^{2} d t\right)^{1 / 2},
$$

and $\mathbf{G} \in \mathcal{Y}_{\text {loc }}$ belongs to the space $\mathbf{Y}_{\gamma}(\mathbb{R})$ consisting of functions satisfying $\mathcal{Q}(t) \mathbf{G}(t)=$ $\mathbf{G}(t)$ for almost all $t \in \mathbb{R}$ and endowed with the norm

$$
\left\|\mathbf{G} ; \mathbf{Y}_{\gamma}(\mathbb{R})\right\|_{\tau}=\left(\int_{\mathbb{R}} e^{-2 \gamma|t-\tau|}\|\mathbf{G}(t) ; Y\|^{2} d t\right)^{1 / 2}
$$

We denote by $\beta$ a positive number such that $\lambda_{1}, \ldots, \lambda_{N}$ are the only eigenvalues of the pencil $\mathcal{A}(\lambda)$ on the intervals $\delta_{\beta^{\prime}}$ with $\left|\beta^{\prime}\right| \leq \beta$.

Lemma 7.1. Let $\gamma \in(0, \beta]$ and $\xi \in \mathbb{R}^{M}, \mathbf{F} \in \mathbf{L}_{\gamma}^{2}(\mathbb{R}), \mathbf{G} \in \mathbf{Y}_{\gamma}(\mathbb{R})$. Then the linear problem (7.27), (7.28) has a unique solution $\mathbf{u} \in H_{\gamma}^{1}(\mathbb{R}), V \in \mathbf{X}_{\gamma}(\mathbb{R})$, and this solution satisfies

$$
\left\|\mathbf{u} ; \mathbf{H}_{\gamma}^{1}(\mathbb{R})\right\|_{\tau} \leq C\left(\|\xi\|+\left\|\mathbf{F} ; \mathbf{L}_{\gamma}^{2}(\mathbb{R})\right\|_{\tau}\right)
$$

and

$$
\left\|V ; \mathbf{X}_{\gamma}(\mathbb{R})\right\|_{\tau} \leq C\left\|\mathbf{G} ; \mathbf{Y}_{\gamma}(\mathbb{R})\right\|_{\tau}
$$

where $C$ is independent of $\tau$. In the case of complex Hilbert spaces we must assume certainly that $\xi \in \mathbb{C}^{M}$.

Given $\gamma$ as in the lemma, we define the linear operator

$$
K: \mathbf{L}_{\gamma}^{2}(\mathbb{R}) \times \mathbb{R}^{M} \times \mathbf{Y}_{\gamma}(\mathbb{R}) \rightarrow H_{\gamma}^{1}(\mathbb{R}) \times \mathbf{X}_{\gamma}(\mathbb{R})
$$

which maps the data $(\mathbf{F}, \xi, \mathbf{G})$ into the solution of the problem (7.27)-(17.28) satisfying the estimates (7.29), (7.30).

Let us sketch the proof of Lemma. By a shift of the $t$-variable the lemma can be reduced to the case $\tau=0$, which is the case we will consider here. Equation (7.27) can be written as

$$
\mathbf{u}(t)=e^{\mathcal{R} t} \xi+\int_{0}^{t} e^{\mathcal{R}(t-s)} \mathbf{F}(s) d s .
$$

Since the eigenvalues $\lambda_{k}, k=1, \ldots, N$, are purely imaginary we have

$$
\left|e^{\mathcal{R} t}\right| \leq C(1+|t|)^{\widehat{m}-1}, \quad \widehat{m}=\max \left\{m_{k, j}: k=1, \ldots, N, j=1, \ldots, J_{k}\right\}
$$

and similar estimate is valid for the $t$-derivative. Therefore

$$
|\mathbf{u}(t)| \leq C\left((1+|t|)^{\widehat{m}-1}+\int_{0}^{t}(1+|t-s|)^{\widehat{m}-1}|\mathbf{F}(s)| d s\right)
$$

and

$$
\left|\mathbf{u}^{\prime}(t)\right| \leq C\left((1+|t|)^{\widehat{m}-1}+\int_{0}^{t}(1+|t-s|)^{\widehat{m}-1}|\mathbf{F}(s)| d s+|\mathbf{F}(t)|\right)
$$

which implies (7.29).

We will use the following version of Theorem 6.1 adapted to our situation.

Theorem 7.2. Let $f \in L_{\mathrm{loc}}^{2}(\mathbb{R} ; Y)$ and

$$
\int_{\mathbb{R}} e^{-\beta|t|}\|f ; \mathcal{Y}(t, t+1)\| d t<\infty
$$


Then, the equation

$$
\mathcal{L}\left(t, D_{t}\right) u=f
$$

has a solution $u=U+V \in \mathcal{X}_{\mathrm{loc}}$ such that $U$ is a solution of (6.1) and $V$ is a solution of (6.2) satisfying the estimate

$$
\|V ; \mathcal{X}(\tau, \tau+1)\| \leq C \int_{\mathbb{R}} e^{-\beta|t-\tau|}\|\mathcal{Q} f ; \mathcal{Y}(t, t+1)\| d t
$$

for all $\tau \in \mathbb{R}$.

Let $f$ satisfy (7.32) and $\mathcal{Q} f=0$. If the bound

$$
\|u ; \mathcal{X}(t, t+1)\| \leq C e^{\beta|t|} \text { for } t \in \mathbb{R}
$$

with some constant $C$ hold for $u$, then $V=0$.

The estimate (7.30) follows from (7.33) by using the arguments in Section 4 of [3; the fact the in the present case the operator $A(t)$ is not constant in $t$ does not influence the proofs. We need to observe that $\beta$ in the reference can be replaced by $\beta_{1}>\beta$ with $\beta_{1}$ so small enough so that the strip $\beta<\Im \lambda \leq \beta_{1}$ does not contain eigenvalues of the pencil $\mathcal{A}(\lambda)$.

7.4. Center manifold reduction. The following theorem is an analog of Theorem 1 in [10] and its proof is literally the same. We present below the main steps of the proof for the convenience of the reader. As in the previous section we consider the real case and hence the matrix $\mathcal{R}$ is real valued and defined in the end of Sect 7.2 .

Theorem 7.3. Suppose that the assumption on $A(t)$ from Sect 2 are valid and the Operator $A(t)$ is real valued. There are neighborhoods of zero $W_{1} \subset \mathbb{R}^{M}, W_{2} \subset X$ and a neighborhood $\mathcal{M}_{0}$ of $\mu_{0}$ in $\mathcal{M}$ and a function

$$
h=h(t, \mu, \xi) \in C^{k}\left(\mathbb{R} \times \mathcal{M}_{0} \times W_{1}, W_{2}\right),
$$

such that

$$
\mathcal{Q}(t) h(t)=h(t), \quad h\left(t, \mu_{0}, 0\right)=0 \quad \text { and } \partial_{\xi} h\left(t, \mu_{0}, 0\right)=0 \text { for } t \in \mathbb{R} .
$$

Moreover the following properties hold:

1. Every solution $\mathbf{u}: \mathbb{R} \rightarrow W_{1}$ of the reduced system

$$
D_{t} \mathbf{u}-\mathcal{R} \mathbf{u}=\mathbf{f}(t, \mu, U(t)+h(t, \mu, \mathbf{u}(t))),
$$

where $\mu \in \mathcal{M}_{0}$ and $U=\mathcal{P} u$ is given by (7.20), gives a solution $u=U+h(t, \mu, \mathbf{u}(t))$ of the whole system (7.1).

2. Every small bounded solution $u: \mathbb{R} \rightarrow X$ of (7.1) satisfying $\mathbf{u}(t) \in W_{1}$ and $V(t) \in W_{2}$ for all $t \in \mathbb{R}$ yields a solution $\mathbf{u}$ of the reduced system (7.34). In this case $V(t)=h(t, \mu, \mathbf{u}(t))$.

The first step of the proof is to study the solvability of the non-linear problem

$$
\begin{aligned}
\left(D_{t}-\mathcal{R}\right) \mathbf{u}(t) & =\mathbf{f}(t, \mu, U(t)+V(t)), \quad \mathbf{u}(\tau)=\xi \\
\mathcal{L}\left(t, D_{t}\right) V(t) & =\mathcal{Q}(t) f(t, \mu, U(t)+V(t))
\end{aligned}
$$

for small $\|\xi\|$. Since we are interested in small solutions it is convenient to introduce new right-hand sides in (7.35), (7.36) which however coincide with the original ones for small $u$. Thus, let

$$
\mathbf{f}_{\varepsilon}(t, \mu, u)=\mathbf{f}(t, \mu, u) \chi\left(\frac{\|\mathbf{u}\|}{\varepsilon}\right) \chi\left(\frac{\|V\|_{X}}{\varepsilon}\right),
$$




$$
f_{\varepsilon}(t, \mu, u)=f(t, \mu, u) \chi\left(\frac{\|\mathbf{u}\|}{\varepsilon}\right) \chi\left(\frac{\|V\|_{X}}{\varepsilon}\right)
$$

where $\chi \in C^{k+1}([0, \infty))$ is a cut-off function such that $\chi(t)=1$ for $t \leq 1$ and $\chi(t)=0$ for $t>2$ and $\varepsilon \in\left(0, \varepsilon_{0}\right)$ with $\varepsilon_{0}$ satisfying

$$
\left\{U+V:\|\mathbf{u}\| \leq 2 \varepsilon_{0},\|V\|_{X} \leq 2 \varepsilon_{0}\right\} \in \widetilde{X} .
$$

Now instead of (7.35), (7.36) we consider the problem

$$
\begin{aligned}
\left(D_{t}-\mathcal{R}\right) \mathbf{u}(t) & =\mathbf{f}_{\varepsilon}(t, \mu, U(t)+V(t)), \quad \mathbf{u}(\tau)=\xi, \\
\mathcal{L}\left(t, D_{t}\right) V(t) & =\mathcal{Q}(t) f_{\varepsilon}(t, \mu, U(t)+V(t)),
\end{aligned}
$$

where $f_{\varepsilon}$ and $\mathbf{f}_{\varepsilon}$ are defined by (7.37). Using the operator $K$ introduced in (7.31), we can write problem (17.39), (7.40) as a fixed point equation

$$
(\mathbf{u}(t), V(t))=K\left(\mathbf{f}_{\varepsilon}(\cdot, \mu, U(\cdot)+V(\cdot)), \xi, \mathcal{Q}(\cdot) f_{\varepsilon}(\cdot, \mu, U(\cdot)+V(\cdot))\right)(t)
$$

Similarly to [10] and using (7.3) among other things, one can show that the operator $K(\cdot, \xi, \cdot)$ is a contraction in a small ball of the Hilbert-space $H_{\gamma}^{1}(\mathbb{R}) \times \mathbf{X}_{\gamma}(\mathbb{R})$ determined by the choices (7.37), (7.38). The problem thus has a unique small solution for small $\varepsilon$. The function $h$ is defined by

$$
h(\tau, \mu, \xi)=V(\tau, \mu, \xi)(\tau) .
$$

The same arguments as in [10] show that $h$ has the required smoothness with respect to $t, \mu$ and $\xi$ and that the properties 1 and 2 hold.

The following remarks can be verified by quite straightforward arguments using the definition of the function $h$ given in the proof of the above theorem.

Remark 7.4. If $f$ is periodic in $t$, then the function $h$ is also periodic in $t$.

Remark 7.5. If additionally

$$
f(t, \mu, 0)=0 \text { for } \mu \in \mathcal{M}, t \in \mathbb{R},
$$

then

$$
h(t, \mu, 0)=0 \text { for } \mu \in \mathcal{M}_{0}, t \in \mathbb{R} \text {. }
$$

Remark 7.6. Let the operator $A(t)$ (and hence $\mathcal{L}$ ) depend also on $\mu \in \mathcal{M}$ of class $C_{\text {per }}^{k+1}(\mathbb{R} \times \mathcal{M} ; L(X, Y))$. So $A=A(t, \mu)$ and $\mathcal{L}\left(t, \mu, D_{t}\right)$. We assume also that the structure of the orthogonal projector $\mathcal{P}=\mathcal{P}(t, \mu)$ is the same for $\mu \in \mathcal{M}$ and it is given by (7.5) where the functions $\varphi_{j m}^{k}=\varphi_{j m}^{k}(t, \mu)$ and $\psi_{j m}^{k}=\psi_{j m}^{k}(t, \mu)$ are smoothly depend on $\mu$ also (of class $C_{\text {per }}^{k+1}(\mathbb{R} \times \mathcal{M} ; X)$ and $C_{\text {per }}^{k+1}(\mathbb{R} \times \mathcal{M} ; Y)$ respectively. If

$$
f(t, \mu, 0)=0 \text { and } \partial_{u} f(t, \mu, 0)=0 \text { for } \mu \in \mathcal{M}, t \in \mathbb{R},
$$

then

$$
h(t, \mu, 0)=0 \quad \text { and } \partial_{\xi} h(t, \mu, 0)=0 \text { for } \mu \in \mathcal{M}_{0} \text { and } t \in \mathbb{R} .
$$

Acknowledgement. V. K. was supported by the Swedish Research Council (VR), 2017-03837. J. T. was supported by a research grant from the Faculty of Science of the University of Helsinki. 


\section{REFERENCES}

[1] Hille, E., Phillips, R., Functional analysis and semi-groups. Amer. Math. Soc., Colloquium publications Vol. 31, Providence RI (1957).

[2] Kondratiev V.A,. Boundary problems for elliptic equations in domains with conical or angular points. Trudy Moskov. Mat. Obshch. 16 (1967), 209292 (English transl. Trans. Moscow Math. Soc. 16 (1967), 227313).

[3] Kozlov, V.A., Maz'ya, V.G., , Differential equations with operator coefficients with applications to boundary value problems for partial differential equations. Springer Monographs in Mathematics. Springer-Verlag, Berlin, 1999.

[4] Kozlov, V.A., Maz'ya, V.G., An asymptotic theory of higher-order operator differential equations with nonsmooth nonlinearities. J. Funct. Anal. 217 (2004), no. 2, 448488.

[5] Kozlov V.A., Mazya V.G., Rossmann J. Elliptic boundary value problems in domains with point singularities. Amer. Math. Soc, Providence RI (1997).

[6] Kuchment P. A. Floquet theory for partial differential equations. Uspehi mat. nauk. 37 (1982), 352 (English transl. Russ. Math. Surveys. 37, 4 (1982), 160).

[7] Kuchment P., Floquet theory for partial differential equations. Birkhäuser, Basel (1993).

[8] Kuchment, P., An overview of periodic elliptic operators. Bull. Amer. Math. Soc. 53, 3 (2016), $343-414$.

[9] Lions, J.-L.; Magenes, E., Non-homogeneous boundary value problems and applications. Vol. I. Translated from the French by P. Kenneth. Die Grundlehren der mathematischen Wissenschaften, Band 181. Springer-Verlag, New York-Heidelberg (1972).

[10] Mielke, Alexander Reduction of quasilinear elliptic equations in cylindrical domains with applications. Math. Methods Appl. Sci. 10 (1988), no. 1, 5166.

[11] Mielke, A., Hamiltonian and Lagrangian flows on center manifolds. With applications to elliptic variational problems. Lecture Notes in Mathematics, 1489, Springer-Verlag, Berlin (1991).

[12] Nazarov, S. A., Elliptic boundary value problems with periodic coefficients in a cylinder. (Russian) Izv. Akad. Nauk SSSR Ser. Mat. v. 45 (1981), 1, 101-112.

[13] Nazarov, S. A., Properties of spectra of boundary value problems in cylindrical and quasicylindrical domains. Sobolev Spaces in Mathematics. V. II (Mazya V., Ed.) International Mathematical Series, Vol. 9, Springer, New York (2008), 261309.

[14] Nazarov, S.A., Plamenevsky, B.A., Elliptic problems in domains with piecewise smooth boundaries. De Gruyter Expositions in Mathematics, 13, Walter de Gruyter \& Co., Berlin (1994). 